Published online: 15 April 2021

https://doi.org/10.1038/s41591-021-01307-8

References

1. PATH. https://www.path.org/media-center/path-announces-

pmi-inform-malaria-operational-research-project/

(10 February 2021).

2. World Health Organization \& RBM Partnership to End Malaria.
High burden to high impact: a targeted malaria response (WHO, 2019). meeting the criteria have been omitted. This letter is signed in an individual capacity. The views and opinions expressed do not necessarily reflect that of any organization they (the authors) are associated with or employed by.

Competing interests

The authors declare no competing interests.

\title{
Africa needs more genome sequencing to tackle new variants of SARS-CoV-2
}

To the Editor-As Africa records new peaks in COVID-19 cases in at least 40 countries, there is evidence linking the new peaks to the emergence of multiple SARS-CoV-2 variants. The World Health Organization Regional Office for Africa has identified a total of 145 lineages, using data from just under 5,000 publicly available sequences from 24 of the 47 countries in the region ${ }^{1}$. On 14 February 2021, the Nigeria Centre for Disease Control reported the detection of 55 different lineages of SARS-CoV-2 circulating in Nigeria ${ }^{2}$, which brought the total number of lineages in Africa to 200 . While that report demonstrates the organizational capacity of next-generation sequencing, the diversity of lineages and the fact that Nigeria contributes over one quarter of the lineages in Africa is worrisome, as it indicates ongoing community spread and accelerated viral mutation. So far, Nigeria has detected 29 cases of the more-transmissible B 1.1.7 variant of SARS-CoV-2. On

18 December 2020, the South African government announced the detection of a new SARS-CoV-2 variant, 501Y.V2, which spread rapidly and displaced other lineages circulating in South Africa ${ }^{3}$. This 501Y.V2 variant has also been reported in Botswana, Comoros, The Gambia, Ghana, Kenya, Mozambique and Zambia. The Africa Centres for Disease Control and Prevention has warned that the rising COVID-19 fatality rates across Africa exceed the current global average of $2.2 \%$ (ref. $^{4}$ ).

New variants are the result of mutations that occur as part of the evolution and adaptation of viruses such as SARS-CoV-2. These mutations distinguish new variants from the wild-type or predominant viral variants already circulating among communities. By altering the spike proteins that are targets of natural and vaccine-induced immunity, new variants of SARS-CoV-2 may become more transmissible and less easy to diagnose and may result in more-severe disease that is less susceptible to therapeutic agents such as monoclonal antibodies. The fact that new variants can potentially evade immunity from natural infection or vaccines is the possibility of greatest concern, as vaccines are the critical new tool in the battle against COVID-19. Africa currently lags behind the global push for mass vaccination against COVID-19. As of 28 February 2021, only eight African countries had commenced vaccination against COVID-19, with under 4 million Africans having received at least one dose of a vaccine against COVID-19 in Morocco (3.6 million), Seychelles $(75,096)$, Algeria $(75,000)$, South Africa $(70,527)$, Senegal $(25,653)$ Zimbabwe $(18,843)$, Mauritius $(3,843)$ and Egypt $(1,315)^{5}$.

Africa is still coming to terms with the results of a recent study indicating that the AstraZeneca vaccine against COVID-19 provides minimal protection against mild to moderate COVID-19 disease resulting from infection with the 501Y.V2 variant, in young South African adults ${ }^{6}$; its efficacy against severe COVID-19 has not been determined. The South African government responded to this by suspending the administration of 1 million doses of the AstraZeneca vaccine already in the country, despite the seeming attractiveness of the vaccine's requiring refrigeration temperatures of $2-8{ }^{\circ} \mathrm{C}$, compared with $-70^{\circ} \mathrm{C}$ for the Pfizer-BioNTech vaccine. Attention has now shifted to the first single-dose Johnson \& Johnson vaccine against COVID-19, which can be stored at the temperature of a regular refrigerator for up to three months. This vaccine was shown to be $57 \%$ effective in preventing moderate to severe COVID19 in clinical trials in South Africa, while newly emerging variants were already in circulation ${ }^{7}$; mild cases of COVID-19 were excluded. The US Food and Drug Administration issued an emergency use authorization for the Johnson \& Johnson vaccine against COVID-19 on 27 February 2021. The first multivalent vaccine was created recently by Moderna in hopes of making it effective against the 501 .V2 variant; this vaccine is going into phase 1 trials soon ${ }^{8}$.

Genomics is a vital part of global surveillance systems and offers huge potential for identifying changes to viruses in real time and for enhancing responses to emerging infectious diseases. The WHO and the Africa Centres for Disease Control and Prevention have jointly established a network of COVID-19 genomic sequencing laboratories across Africa in the Democratic Republic of Congo, The Gambia, Kenya, Nigeria, Senegal, South Africa and Uganda'

In our view, public-private partnerships (PPPs) involving national governments, academic institutions and public-health institutions are needed for realization of the full potential of genomics in Africa. The Network for Genomic Surveillance in South Africa consortium, which sequenced 1,365 genomes and identified 16 new lineages of SARS-CoV-2 in 2020 (ref. ${ }^{10}$ ), is an example of a successful local PPP initiative worth promoting. Substantial investment is required from regional PPPs for further boosting of research infrastructure and capacity development for genomics. While the Africa Centres for Disease Control and Prevention is playing a vital role in building genomics-based surveillance systems and reinforcing collaborative networks, national governments will need to provide regulatory frameworks for genomics in Africa.

The emergence of new variants of SARS-CoV-2 offers an opportunity for strengthening the involvement of the Africa Pathogen Genomics Initiative, launched in October 2020; for consolidating 
next-generation sequencing capacity in Africa; and for accelerating surveillance and emergency responses for better public-health outcomes. Only then will the world achieve uhuru-freedom.

Akaninyene Otu ${ }^{1,2,5}{ }^{凶}$, Emmanuel Agogo $^{3}$ and Bassey Ebenso ${ }^{4,5}$

${ }^{1}$ Department of Infection and Travel Medicine, Leeds Teaching Hospitals NHS Trust, Leeds, UK. ${ }^{2}$ College of Medical Sciences, University of Calabar, Calabar, Nigeria. ${ }^{3}$ Resolve to Save Lives, Abuja, Nigeria. ${ }^{4}$ Leeds Institute of Health Sciences, University of Leeds, Leeds, UK. ${ }^{5}$ These authors contributed equally: Akaninyene Otu, Bassey Ebenso.

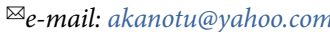

Published online: 7 April 2021

https://doi.org/10.1038/s41591-021-01327-4

References

1. World Health Organization Regional Office for Africa. https://www.afro.who.int/news/seven-things-know-about-covid19-variants-africa (22 January 2021)

2. Nigeria Centre for Disease Control. https://ncdc.gov.ng/ news/322/statement-on-variants-of-sars-cov-2-in-nigeria (19 February 2021).

3. World Health Organization. https://www.who.int/csr/don/31 december-2020-sars-cov2-variants/en/ (31 December 2020).

4. Africa Centres for Disease Control and Prevention. https://africacdc.org/news-item/statement-on-the-efficacyof-the-astrazeneca-covid-19-vaccine-chadox1-ncov-19-againstthe-sars-cov-2-variants/ (10 February 2021).

5. Our World in Data. https://ourworldindata.org/covidvaccinations (accessed 1 March 2021).

6. Madhi, S. A. et al. N. Engl. J. Med. https://doi.org/10.1056/ NEJMoa2102214 (2021)
7. Johnson \& Johnson. https://www.jnj.com/johnson-johnsonannounces-single-shot-janssen-covid-19-vaccine-candidatemet-primary-endpoints-in-interim-analysis-of-its-phase3-ensemble-trial (accessed March 2021).

8. Financial Times. https://www.ft.com/content/al4a91b1-8ced45ef-b679-1f2bada4f50b (accessed March 2021)

9. World Health Organization Regional Office for Africa. https://www.afro.who.int/news/new-covid-19-variants-f uelling-africas-second-wave (27 February 2021).

10. Tegally, H. et al. Nat. Med. 27, 440-446 (2021).

Author contributions

B.E. and A.O. conceived of the paper; B.E. and A.O. wrote the original draft of the manuscript with contributions from E.A.; and all authors reviewed and approved the final version of the manuscript.

Competing interests

The authors declare no competing interests. 